\title{
Can preoperative serum thyroglobulin and anti- thyroglobulin levels predict malignant potential of a thyroid nodule with atypia or follicular lesion of undetermined significance?
}

\author{
Amal Alhefdhi ${ }^{1,2 *}$, Tahani Altayyar ${ }^{3}$, Saad M Alqahtani ${ }^{4}$, Mohammed Alshehri ${ }^{5}$, Salahudin Elnaas ${ }^{6}$ and Saif Alsobhi $^{1,2}$ \\ ${ }^{1}$ Department of General Surgery, Breast and Endocrine Section, King Faisal Specialist Hospital \& Research Center (KFSH\&RC), Riyadh, Saudi Arabia \\ ${ }^{2}$ Alfaisal University, Riyadh, Saudi Arabia \\ ${ }^{3}$ Department of Surgery, King Abdulaziz Hospital, Ministry of Health,Makkah,Saudi Arabia \\ ${ }^{4}$ Department of Surgery, College of Medicine, Majmaah University, Majmaah 11952, Saudi Arabia \\ ${ }^{5}$ Department of Surgery, King Saud Medical City, Riyadh, Saudi Arabia \\ ${ }^{6}$ Department of Radiology, King Faisal Specialist Hospital \& Research Center (KFSH\&RC), Riyadh, Saudi Arabia
}

\begin{abstract}
Background: The heterogeneity of atypia of undetermined significance (AUS) and follicular lesion of undetermined significance (FLUS) renders it difficult to classify the risk as benign, suspicious, or malignant. In the present study, we assessed whether patients' preoperative demographic, radiological, and biochemical characteristics can predict the postoperative pathological diagnosis in patients with AUS/FLUS.

Methods: Retrospective review of patients with AUS/FLUS who underwent surgery over a six-year period was conducted. The primary outcome was prediction of the malignant potential of AUS/FLUS nodules based on demographic data, thyroid imaging reporting and data system (TIRADS) score, and thyroglobulin (Tg) level.

Results: Fifty-one cases were identified with a mean age of $40 \pm 12$ years and 36 (70.6\%) were female. The final pathological diagnosis of malignancy was seen in 29 patients (56.9\%). Patients with malignancy had a higher Tg level and TIRADS points $(154 \pm 167 \mathrm{vs.} 463 \pm 733 \mu \mathrm{g} / \mathrm{L}, \mathrm{p}=0.0352$ and $3.3 \pm 1.4$ vs. $5.9 \pm 2.7, \mathrm{p}=$ 0.0001 , respectively). The final diagnosis did not differ by patient demographics (age and gender).

Conclusion: AUS/FLUS nodules in patients with high Tg level and TIRADS score showed a higher risk of malignancy, which was observed in nearly $50 \%$ of the patients in this retrospective study. Therefore, preoperative $\mathrm{Tg}$ and high TIRADS score could be useful in malignancy-risk stratification in thyroid nodules with indeterminate cytology. Further prospective multicenter studies that may throw light on this common endocrine cancer among Saudis and specifically among women, are needed to confirm our findings.
\end{abstract}

\begin{abstract}
Abbreviations: ACR: American College of Radiology; ATA: American Thyroid Association; AUS: Atypia of Undetermined Significance; BSRTC: Bethesda System for Reporting Thyroid Cytopathology; FLUS: Follicular Lesion of Undetermined Significance; FNAB: Fine-Needle Aspiration Biopsy; FNAC: Fine-Needle Aspiration Cytology; KFSH\&RC: King Faisal Specialist Hospital \& Research Center; PTC: Papillary Thyroid Cancer; TIRADS: Thyroid Imaging Reporting and Data System; US: Ultrasound
\end{abstract}

\section{Introduction}

Thyroid cancer is the most common endocrine cancer worldwide [1], and in Saudi Arabia [2,3], where it accounts for approximately 9\% of all malignancies and $12 \%$ of female malignant cancers [4]. Thyroid cancer is considered the second most common cancer among Saudi women, and the fourth among Saudi men [4]. Thyroid nodules are the most common presentation of thyroid cancer [5]. However, thyroid nodules are highly prevalent, accounting for $20 \%-76 \%$ in the general population [6]. Since therapeutic management of malignant nodules is different from that of benign nodules, it is vital to distinguish the two $[5,6]$.
The Thyroid Imaging, Reporting, and Data System (TIRADS) published by the American College of Radiology (ACR) in 2015 is an ultrasonographic, risk-stratification system in which the nodules are scored for cancer risk based on composition, echogenicity, shape, margin, and echogenic foci by ultrasound (US) [7] and assigned to one of five malignancy-risk groups; reported cancer risks of $0.3 \%$ (TR1benign), $1.5 \%$ (minimally suspicious-TR2), $4.8 \%$ (mildly suspiciousTR3), 5\%-20\% (moderately suspicious-TR4) and 35\% (highly suspicious-TR5) [7].

In addition, the 2015 American Thyroid Association (ATA) Management Guidelines for Adult Patients with Thyroid Nodules and Differentiated Thyroid Cancer, reinforced the importance of the

*Correspondence to: Amal Alhefdhi, Department of General Surgery, Breast and Endocrine Section, King Faisal Specialist Hospital \& Research Center (KFSH\&RC), Riyadh, Saudi Arabia, Tel: +966 11 4427755, Fax: +966 11 4427772; E-mail: alhefdhi@kfshrc.edu.sa

Key words: thyroid imaging, follicular lesion, malignancy risk, thyroid nodules

Received: April 03, 2020; Accepted: April 20, 2020; Published: April 24, 2020 
Alhefdhi A (2020) Can preoperative serum thyroglobulin and anti-thyroglobulin levels predict malignant potential of a thyroid nodule with atypia or follicular lesion of undetermined significance?

fine-needle aspiration cytology (FNAC) as the initial screening test for thyroid nodules [8]. The Bethesda System for Reporting Thyroid Cytopathology (BSRTC) classifies thyroid FNAC cytology into six malignancy-risk groups: reported cancer risks of $1 \%-4 \%$ (nondiagnostic category-DC I), 0\%-3\% (benign-DC II), 5\%-15\% (atypia/follicular lesion of undetermined significance-DC III), 15\%-30\% (follicular neoplasm/suspicion for a follicular neoplasm-DC IV), $60 \%-75 \%$ (suspicious for malignancy-DC V) and 97\%-99\% (malignant-DC VI) [9].

Based on the ACR recommendations, FNAC is not required for TR1 and TR2 nodules, but it is mandatory for TR3, TR4, and TR5 nodules if they measure $2.5,1.5$, and $1.0 \mathrm{~cm}$, respectively [10]. Although, both ACR and ATA do not recommend the FNAC for nodules that measure $<1.0 \mathrm{~cm}[6,8]$, FNAC is justified in nodules measuring 5 to $9 \mathrm{~mm}$ that fall under risk category $[7,9]$.

Atypia of Undetermined Significance (AUS) or Follicular Lesion of Undetermined Significance (FLUS) is considered the third BSRTC category. The risk of malignancy in this particular group is 5\%-15\% [911], but because it is a heterogeneous group, categorizing AUS into the six malignancy-risk groups is difficult. To investigate AUS further, it is essential to identify a suitable biomarker, which remains a challenge. To predict malignancy in thyroid nodules, some have used molecular, cytological/morphologic and imaging, whereas others have focused on the levels of preoperative thyroglobulin $(\mathrm{Tg})$, albeit with conflicting results [12]. Normal or well-differentiated malignant thyrocytes produce $\mathrm{Tg}$, a glycoprotein that may be used as tumour marker for patients with differentiated thyroid cancer (DTC) [12].

Genetic testing of the thyroid fine-needle aspiration biopsy (FNAB) detects malignancy in patients with AUS/FLUS with high sensitivity and specificity $[8,13,14]$. Since all tertiary hospitals do not provide this genetic testing, the need for an alternative method is warranted. This study aimed to describe the correlation between preoperative demographic data, radiological characteristics required for identifying TIRADS-based malignancy-risk groups, biochemical characterization of $\mathrm{Tg}$ and anti-Tg antibody and final postoperative pathological diagnosis among patients with AUS/FLUS.

\section{Methods}

This study was approved by the Office of Research Affairs at King Faisal Specialist Hospital \& Research Center (KFSH\&RC), Riyadh, Saudi Arabia. Institutional review board approval number 2161182. Between 2011 and 2016, adult patients with primary diagnosis of AUS/FLUS and who underwent surgery at the King Faisal Specialist Hospital \& Research Center (KFSH\&RC), Riyadh, Saudi Arabia, and who had documented preoperative $\mathrm{Tg}, \mathrm{TgAb}$ and $\mathrm{TSH}$ levels, were included in this retrospective study, which comprised patients with a final diagnosis of both benign and malignant tumours. We excluded pediatrics cases (younger than 18 years old) and patients with missing records, and those who were operated at an external hospital. All cases had repeated FNAC with two consecutive results of AUS or FLUS on cytology. Patient data including age, gender, TSH, Tg level, Tg antibody $\mathrm{Ab}$ ) and US variables using the TIRADS score were retrieved from the electronic medical records. Missing variables were obtained from the patients' charts.

All images were reviewed and scored by one radiologist. We used the ACR TIRADS points based on the criteria published in 2017 [15]. Scoring was based on the nodule's composition ( 0 for cystic or completely cystic or spongiform, 1 for mixed cystic and solid or solid or almost completely solid); echogenicity ( 0 for anechoic, 1 for hyper- or isoechoic, 2 for hypoechoic, and 3 for very hypoechoic); shape (0 for wider than tall, 3 for taller than wide); margin ( 0 for smooth or illdefined, 2 for lobulated/irregular, and 3 for extra-thyroidal extension); echogenic foci ( 0 for none or large comet tail artifact, 1 for macro calcifications, 2 for peripheral/rim calcifications, and 3 for punctate echogenic foci). The TIRADS groups were allocated based on the sum of points: TR1: 0; TR2: 2; TR3: 3; TR4: 4-6; and TR5: $\geq 7$ points [15]. On the basis of the TIRADS points, malignancy of the thyroid was classified as: normal thyroid gland (TIRADS 1), benign lesions (TIRADS 2), probably benign lesions (TIRADS 3), suspicious lesions (TIRADS 4), probably malignant lesions (TIRADS 5) and biopsyproven malignancy (TIRADS 6) [16,17].

All statistical analyses were performed using SAS version 9.4 (SAS Institute Inc., Cary, NC, USA). Descriptive statistics for the continuous variables were reported as mean \pm standard deviation and categorical variables were summarized as frequencies and percentages. The continuous variables were compared using Student's independent t-test, Pearson correlation test, and scatter diagram. The categorical variables were compared by Chi-square test. The level of statistical significance is set at $\mathrm{p}<0.05$.

\section{Results}

In this retrospective analysis, we screened 161 cases of AUS/FLUS. After applying the inclusion and exclusion criteria, 51 cases met our study criteria in which the patients had documented preoperative serum Tg, TgAb, and TSH levels. Patients' mean age was $40 \pm 12$ years. In general, the final pathological diagnosis of malignancy was noted in 29 patients (56.9\%), while the remaining (43.1\%) were benign. Females $(\mathrm{n}=36)$ comprised $71 \%$ of the total. Though malignancy rate $(73.3 \%)$ was higher among males, it was not statistically significant $(p=0.2142)$. Patient demographic characteristics did not differ by tumour status (Table 1).

The mean serum $\mathrm{Tg}$ and $\mathrm{TgAb}$ levels were $330 \pm 580 \mu \mathrm{g} / \mathrm{L}$ and 153 $\pm 583 \mathrm{U} / \mathrm{ml}$ retrospectively, while the mean TSH level was $1.84 \pm 1.2$ $\mathrm{mU} / \mathrm{L}$. The mean serum Tg was significantly higher among patients with malignancy compared with patients with benign tumours (463 \pm $733 \mu \mathrm{g} / \mathrm{L} v s .154 \pm 167 \mu \mathrm{g} / \mathrm{L} ; \mathrm{p}=0.035$ respectively). The mean TgAb and TSH did not differ by tumour status (Table 1).

Of the 51 patients, 49 had available US images that were reexamined by a radiologist using the TIRADS score. Reports for two cases were unavailable. The TIRADS points were higher among patients with malignancy compared with those with benign tumours $(5.9 \pm$ 2.7 vs. $3.3 \pm 1.4 ; \mathrm{p}=0.0001$ respectively). The mean size of the largest thyroid nodule was $38 \pm 24 \mathrm{~mm}$, but the mean size of the nodule did not differ by tumour status (Table 1).

There were 46 (90.2\%) cases with TIRADS $\geq 3$, of which 27 (52.9\%) had thyroid cancer in the final pathology, and were distributed as

Table 1. Characteristics of patients with AUS/FLUS by tumour status

\begin{tabular}{|l|c|c|c|}
\hline $\begin{array}{l}\text { Total } \\
\mathbf{N}=51\end{array}$ & $\begin{array}{c}\text { Benign } \\
\mathbf{N}=22\end{array}$ & $\begin{array}{c}\text { Malignant } \\
\mathbf{N}=29\end{array}$ & P value \\
\hline Age (years) & $41 \pm 11$ & $40 \pm 14$ & 0.7523 \\
\hline Gender & $4(27.7 \%)$ & $11(73.3 \%)$ & 0.2142 \\
Male $15(29.4 \%)$ & $18(50 \%)$ & $18(50 \%)$ & \\
\hline Female 36(70.6\%) & $1.95 \pm 1.4$ & $1.75 \pm 1.3$ & 0.5644 \\
\hline TSH (mU/L) & $154 \pm 167$ & $463 \pm 733$ & $\mathbf{0 . 0 3 5 2}$ \\
\hline Tg level $(\mu \mathrm{g} / \mathrm{L})$ & $93 \pm 197$ & $199 \pm 757$ & 0.4758 \\
\hline TgAb (U/ml) & $37 \pm 20$ & $40 \pm 26$ & 0.6949 \\
\hline US size biggest nodule $(\mathrm{mm})$ & $3.3 \pm 1.4$ & $5.9 \pm 2.7$ & $\mathbf{0 . 0 0 0 1}$ \\
\hline Overall TIRADS Points & & & \\
\hline
\end{tabular}


Alhefdhi A (2020) Can preoperative serum thyroglobulin and anti-thyroglobulin levels predict malignant potential of a thyroid nodule with atypia or follicular lesion of undetermined significance?

follows: 23 cases with TIRADS 3 of which nine (39\%) had thyroid cancer (four follicular cancer and five papillary thyroid cancer (PTC); two with follicular variant); 12 cases with TIRADS 4 of which eight (66.7\%) had thyroid cancer (two with follicular cancer and six with PTC; three with follicular variant); 11 cases with TIRADS 5 of which 10 (90.9\%) had thyroid cancer (two with follicular cancer and eight with PTC; one with follicular variant). This is shown in table 2 and graphically depicted in figure 1.

\section{Discussion and conclusion}

Examination of the correlation between preoperative demographic data, radiological characteristics required for identifying TIRADSbased malignancy-risk groups, biochemical characterization of $\mathrm{Tg}$ and anti-Tg antibody and final postoperative pathological diagnosis among patients with AUS/FLUS showed that AUS/FLUS nodules in patients with high Tg level and TIRADS score showed a higher risk of malignancy, which was observed in nearly $50 \%$ of the patients in this retrospective study.

Age and gender were not significant variables influencing the differences between benign and malignant groups in this study, and are in line with previous studies $[18,19]$. However, the risk of malignancy of $56.9 \%$ was higher than most published studies, which ranged from $13.5 \%$ to $43 \%[11,20,21]$, but is in concordance with a recent study, which found a $58.1 \%$ rate of malignancy in AUS/FLUS nodules [18].

We found higher preoperative serum levels of $\mathrm{Tg}$ among patients with thyroid malignancy, suggesting that higher Tg level could increase the risk of malignancy. This finding was in concordance with Sands

Table 2. Comparison of TIRADS score and final pathological diagnosis among patients with AUS/FLUS. Note: Total cases, 49. We excluded two cases since the US was performed at an external hospital and was not reviewed by the radiologist at the time of the study. NA: not available

\begin{tabular}{|l|l|l|l|l|}
\hline $\begin{array}{l}\text { TIRADS } \\
\text { Points/Scor }\end{array}$ & No. & Benign & Cancer (\%) & Type of cancers \\
\hline 1/TR2 & 2 & 2 & $0(0 \%)$ & No cancer \\
\hline 2/TR2 & 1 & 1 & $0(0 \%)$ & No cancer \\
\hline 3/TR 3 & 23 & 14 & $9(39 \%)$ & $\begin{array}{l}4 \text { follicular cancer } \\
5 \text { papillary cancer, } 2 \text { of them with follicular } \\
\text { variant }\end{array}$ \\
\hline 4/TR4 & 3 & 2 & $1(33.3 \%)$ & 1 papillary cancer, follicular variant \\
\hline 5/TR4 & 1 & 1 & $0(0 \%)$ & NA but documented to be benign \\
\hline 6/TR4 & 8 & 1 & $7(87.5 \%)$ & $\begin{array}{l}5 \text { papillary cancer, 3 of them with follicular } \\
\text { variant } \\
\text { f follicular cancer }\end{array}$ \\
\hline 7/TR5 & 6 & 1 & $5(83.3 \%)$ & $\begin{array}{l}3 \text { papillary cancer } \\
\text { 2 follicular cancer }\end{array}$ \\
\hline 7/TR5 & 5 & 0 & $5(100 \%)$ & $\begin{array}{l}5 \text { papillary cancer, 1 of them with follicular } \\
\text { variant }\end{array}$ \\
\hline
\end{tabular}

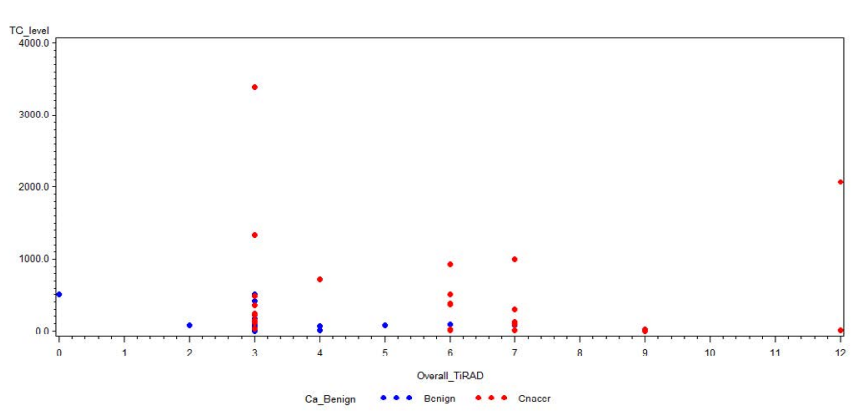

Figure 1. Scatter Diagram showing relationship between TIRADS score and pathological diagnosis in patients with AUS and FLUS. Note: Source: KFSH\&RC (2011-2016). et al. [22] who reported that high presurgical serum Tg levels may be predictive of well-differentiated thyroid cancer, thereby aiding in the management of nodules with indeterminate cytology. Further, Besic and Besic stated that preoperative Tg and gender were independent predictors of malignancy in follicular or Hürthle cell neoplasms [23].

Petric et al. [24] reported that patient age, solitary tumour, and preoperative serum level of $\mathrm{Tg}$ were independent predictors of malignancy in follicular neoplasms. Strazisar et al. [25] reported that patient age and preoperative serum level of $\mathrm{Tg}$ were independent predictors of malignancy in Hürthle cell neoplasms. In a systematic review of 13 studies, with 9 that focused on thyroid nodules with indeterminate cytology, found a significance difference between benign and malignant nodules in association with mean or median serum preoperative $\mathrm{Tg}$ level [12]. Therefore, we concluded Tg to be an independent predictor of thyroid cancer, particularly with indeterminate cytology [12].

Though we could not detect a significant difference in the preoperative serum level of $\mathrm{TgAb}$ between benign and malignant groups, Karatzas et al. [26] found that in thyroid nodules with indeterminate cytology, there was a significant correlation between the TgAb and PTC $(\mathrm{P}<0.001)$. Hosseini et al. [27] found that high TgAb level $(\geq 30 \mathrm{IU} / \mathrm{ml})$ may increase the risk of malignancy. On the contrary, Kim et al. [28] reported that a positive TgAb test is an independent predictor of thyroid nodule malignancy along with TSH results, regardless of autoimmune thyroiditis. In contrast, Yalcin et al. [29] and Yazici et al. [30] found that high preoperative serum levels of serum Tg, anti-TPO, and $\mathrm{TgAb}$, and high preoperative serum TSH levels were not predictive in thyroid cancer.

All patients with AUS/FLUS in our study with a thyroid malignancy had TIRADS 3 or more, and subsequently higher overall TIRADS points increase the risk of malignancy, which was implied from the risk of malignancy in TIRADS 3 (39\%), 4 (66.7\%), and 5 (90.9\%), which is in agreement with the reported risks of cancer for the same category: $5 \%, 50 \%-80 \%$, and $>80 \%$, respectively [17], with the caveat that these rates were based on the risk of malignancy using the TIRADS score in general. In this study, the TIRADS score was used only for AUS/FLUS group.

A previous report showed that US variables and TIRADS categories did not differ between benign and malignant nodules or between malignancies with one and two AUS/ FLUS nodules [18]. Park et al. [18] assessed the rate of malignancy and characteristics of thyroid nodules in 31 patients with AUS/FLUS and found a high (58.1\%) rate of malignancy with no differences between the benign and malignant nodules in demographic or US characteristics.

Thus, AUS/FLUS nodules in patients with high Tg level and TIRADS score showed a higher risk of malignancy. This study is not without its limitations. The retrospective nature of the study and the small sample size limit the generalizability of our findings. Thus, we use it as a pilot study, and we are considering a prospective multicentre clinical study to confirm our findings.

In conclusion, elevated preoperative Tg and high TIRADS score could be useful in malignancy-risk stratification in thyroid nodules with indeterminate cytology. The limitations of this study can be overcome by pursuing prospective multicentre studies that may throw light on this common endocrine cancer among Saudis and specifically among women. 
Alhefdhi A (2020) Can preoperative serum thyroglobulin and anti-thyroglobulin levels predict malignant potential of a thyroid nodule with atypia or follicular lesion of undetermined significance?

\section{Acknowledgment}

We thank Mr. Abdelmoneim M. Eldali, MSc. (King Faisal Specialist Hospital, Research Centre, Department of Biostatistics, Epidemiology and Scientific Computing) for his assistance in conducting the statistical analysis for this study.

\section{References}

1. Mao Y, Xing M (2016) Recent incidences and differential trends of thyroid cancer in the USA. Endocr Relat Cancer 23: 313-22.

2. Hussain F, Iqbal S, Mehmood A, Bazarbashi S, ElHassan T, et al. (2013) Incidence of thyroid cancer in the Kingdom of Saudi Arabia, 2000-2010. Hematol Oncol Stem Cell Ther 6:58-64.

3. (2014) Cancer Incidence Report Saudi Arabia 2010. Thyroid Cancer.

4. King Faisal Specialist Hospital Tumor Registry Annual Report 2010.

5. Weiss VL, Andreotti RF, Ely KA (2018) Use of the thyroid imaging, reporting, and data system (TIRADS) scoring system for the evaluation of subcentimeter thyroid nodules. Cancer Cytopathol 126: 518-524. [Crossref]

6. Yoon JH, Han K, Kim EK, Moon HJ, Kwak JY (2017) Diagnosis and management of small thyroid nodules: a comparative study with six guidelines for thyroid nodules. Radiology 283: 560-599. [Crossref]

7. Middleton WD, Teefey SA, Reading CC, Langer JE, Beland MD, et al. (2017) Multiinstitutional analysis of thyroid nodule risk stratification using the American College of Radiology Thyroid Imaging Reporting and Data System. AJR Am J Roentgenol 208: 1331-1341. [Crossref]

8. Haugen BR, Alexander EK, Bible KC, Doherty GM, Mandel SJ, et al. (2016) 2015 American Thyroid Association management guidelines for adult patients with thyroid nodules and differentiated thyroid cancer: the American Thyroid Association guidelines task force on thyroid nodules and differentiated thyroid cancer. Thyroid 26: 1-133. [Crossref]

9. Cibas ES, Ali SZ (2009) The Bethesda system for reporting thyroid cytopathology. Am J Clin Pathol 132: 658-665.

10. Çuhaci N, Arpaci D, Üçler R, Yazgan AK, Kıyak G, et al. (2014) Malignancy rate of thyroid nodules defined as follicular lesion of undetermined significance and atypia of undetermined significance in thyroid cytopathology and its relation with ultrasonographic features. Endocr Pathol 25: 248-256. [Crossref]

11. Broome J, Cate F, Solorzano C (2014) Utilization and impact of repeat biopsy for follicular lesion/atypia of undetermined significance. World J Surg 38: 628-633. [Crossref]

12. Trimboli P, Treglia G, Giovanella L (2015) Preoperative measurement of serum thyroglobulin to predict malignancy in thyroid nodules: a systematic review. Horm Metab Res 47: 247-252. [Crossref]

13. Labourier E, Shifrin A, Busseniers AE, Lupo MA, Manganelli ML, et al. (2015) Molecular testing for miRNA, mRNA, and DNA on fine-needle aspiration improves the preoperative diagnosis of thyroid nodules with indeterminate cytology. J Clin Endocrinol Metab 100: 2743-2750. [Crossref]

14. Nikiforov YE, Carty SE, Chiosea SI, Coyne C, Duvvuri U, et al. (2015) Impact of the multi-gene ThyroSeq next-generation sequencing assay on cancer diagnosis in thyroid nodules with atypia of undetermined significance/follicular lesion of undetermined significance cytology. Thyroid 25: 1217-23. [Crossref]
15. Tessler FN, Middleton WD, Grant EG, Hoang JK, Berland LL, et al. (2017) ACR Thyroid Imaging, Reporting and Data System (TIRADS): White paper of the ACR TIRADS Committee. J Am Coll Radiol 14: 587-595. [Crossref]

16. Singaporewalla R, Hwee J, Lang T, Desai V (2017) Clinico-pathological correlation of thyroid nodule ultrasound and cytology using the TIRADS and Bethesda classifications. World J Surg 41: 1807-1811. [Crossref]

17. Vargas-Uricoechea H, Meza-Cabrera I, Herrera-Chaparro J (2017) Concordance between the TIRADS ultrasound criteria and the BETHESDA cytology criteria on the nontoxic thyroid nodule. Thyroid Res 10: 1. [Crossref]

18. Park VY, Kim EK, Kwak JY, Yoon JH, Moon HJ (2015) Malignancy risk and characteristics of thyroid nodules with two consecutive results of atypia of undetermined significance or follicular lesion of undetermined significance on cytology. Eur Radiol 25: 2601-2607. [Crossref]

19. Teixeira GV, Chikota H, Teixeira T, Manfro G, Pai SI, Tufano RP (2012) Incidence of malignancy in thyroid nodules determined to be follicular lesions of undetermined significance on fine-needle aspiration. World J Surg 36: 69-74. [Crossref]

20. VanderLaan PA, Marqusee E, Krane JF (2011) Clinical outcome for atypia of undetermined significance in thyroid fine-needle aspirations: should repeated fna be the preferred initial approach? Am J Clin Pathol 135: 770-775. [Crossref]

21. Ho AS, Sarti EE, Jain KS, Wang H, Nixon IJ, et al. (2014) Malignancy rate in thyroid nodules classified as Bethesda category III (AUS/FLUS). Thyroid 2014;24: 832-839. [Crossref]

22. Sands NB, Karls S, Rivera J, Tamilia M, Hier MP, et al. (2010) Preoperative serum thyroglobulin as an adjunct to fine-needle aspiration in predicting well-differentiated thyroid cancer. J Otorhinolaryngol Head Neck Surg 39: 669-673. [Crossref]

23. Besic H, Besic N (2014) Preoperative serum thyroglobulin concentration as a predictive factor of malignancy in small follicular and Hürthle cell neoplasms of the thyroid gland. World J Surg Oncol 12: 282. [Crossref]

24. Petric R, Perhavec A, Gazic B, Besic N (2012) Preoperative serum thyroglobulin concentration is an independent predictive factor of malignancy in follicular neoplasms of the thyroid gland. J Surg Oncol 105: 351-356. [Crossref]

25. Strazisar B, Petric R, Sesek M, Zgajnar J, Hocevar M, et al. (2010) Predictive factors of carcinoma in 279 patients with Hürthle cell neoplasm of the thyroid gland. $J$ Surg Oncol 101: 582-586.

26. Karatzas T, Vasileiadis I, Zapanti E, Charitoudis G, Karakostas E, et al. (2016) Thyroglobulin antibodies as a potential predictive marker of papillary thyroid carcinoma in patients with indeterminate cytology. Am J Surg 2016 212: 946-952. [Crossref]

27. Hosseini S, Payne R, Zawawi F, Mlynarek A, Hier M, Tamilia M, et al. (2016) Can preoperative thyroglobulin antibody levels be used as a marker for well differentiated thyroid cancer? J Otorhinolaryngol Head Neck Surg 45: 31. [Crossref]

28. Kim ES, Lim DJ, Baek KH, Lee JM, Kim MK, et al. (2010) Thyroglobulin antibody is associated with increased cancer risk in thyroid nodules. Thyroid 20: 885-891. [Crossref]

29. Yalcin S, Ülger BV, Parlak Ö, Ucar AE, Sarikaya SM, et al. (2011) The role of preoperative serum thyroglobulin and thyroid auto-antibody levels before histopathological diagnosis of thyroid cancers. Turk J Med Sci 41: 487-493.

30. Yazici P, Mihmanli M, Bozkurt E, Ozturk FY, Uludag M (2016) Which is the best predictor of thyroid cancer: thyrotropin, thyroglobulin or their ratio? Hormones 15 : 256-263. [Crossref]

Copyright: (C2020 Alhefdhi A. This is an open-access article distributed under the terms of the Creative Commons Attribution License, which permits unrestricted use, distribution, and reproduction in any medium, provided the original author and source are credited. 